\title{
Variable Stiffness Control for Oscillation Damping
}

\author{
G. M. Gasparri ${ }^{\dagger}$, M. Garabini ${ }^{\dagger}$, L. Pallottino ${ }^{\dagger}$, L. Malagia ${ }^{\dagger}$, M. Catalano ${ }^{\dagger \ddagger}$, G. Grioli $^{\dagger \ddagger}$ and A. Bicchi ${ }^{\dagger \dagger}$
}

\begin{abstract}
In this paper a model-free approach for damping control of Variable Stiffness Actuators is proposed. The idea is to take advantage of the possibility to change the stiffness of the actuators in controlling the damping. The problem of minimizing the terminal energy for a one degree of freedom spring-mass model with controlled stiffness is first considered. The optimal bang-bang control law uses a maximum stiffness when the link gets away from the desired position, i.e. the link velocity is decreasing, and a minimum one when the link is going towards it, i.e. the link velocity is increasing. Based on Lyapunov stability theorems the obtained law has been proved to be stable for a multi-DoF system. Finally, the proposed control law has been tested and validated through experimental tests.
\end{abstract}

\section{INTRODUCTION}

Soft Robotics [1] is becoming more and more popular in the research community since it has been proved that the introduction of fixed or adjustable compliant and/or damping elements substantially increases the robot performance. Indeed, at least three robot characteristics take advantages by the introduction of passive elasticity in the robot design: robustness, energy efficiency and peak performance. The robustness against unpredictable impacts can be increased as the passive compliance acts as a low-pass filter cutting the peak forces and preserving the gearboxes life [1]. The possibility to store and release energy into the springs can be exploited to increase the energy efficiency of Soft Robots in cyclic motions (see e.g. [2], [3]). Optimal control studies ([4], [5] and [6]) showed how the peak speed of a conventional motor can be more than doubled if a spring of proper stiffness is used.

Several soft actuation concept have been presented (for an updated review see e.g. [7] and [8]): from Series Elastic Actuation (SEA) [9] to Variable Stiffness Actuation (VSA) [10] to actuators in which it is possible to vary both stiffness and damping at physical level [11]. In more recent years, complex multi-joint Soft Robots have been presented such as the DLR Hand Arm System [12] and the CompActTM arm [13], or the ready to use and low cost qbmove platform based on the VSA Cube [10].

In Soft Robots, on one hand, it is desirable to have the joint damping as small as possible for i) maximising efficiency in cyclic tasks and ii) allowing highly dynamic motion. On the other hand, a low damping value poses challenges if a precise tracking in point-to-point motions is required. Hence it is desiredable having a variable damping in softrobots. A

\footnotetext{
† Centro Int. di Ricerca E. Piaggio, Univ. of Pisa, 56126 Pisa, Italy. Italian Inst. of Technologies, Adv. Robotics, 16163 Genova, Italy. This work is supported by the EC under the grant agreements no.611832 Walk-Man and ICT-287513 "SAPHARI".
}
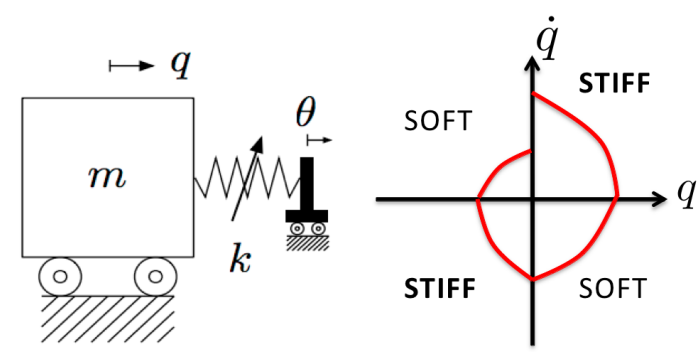

Fig. 1: Left: schematic of a 1 DoF soft actuator used for the optimal control problem. Right: Optimal stiffness switching control provided in this work.

solution to the problem of having variable damping is to physically add, in the robot design, the possibility to adjust the damping (see e.g. [11] , [13]) at the cost of increasing the complexity and the weight of the structure. An alternative approach is the active damping control as the one proposed in this work.

Several solutions for damping control have been implemented in the literature such as: feedback linearization [14], linear-quadratic regulators [15], learning control schemes [16], or modal state-based feedback controllers [17]. A drawback of these approaches is the requirement of a model of the robot. In case of Soft Robots this leads to the necessity of a model identification process that, given the complex dynamics of the system, is a challenging and time-consuming task. Moreover, the model representation itself is prone to imprecisions. Finally, these approaches (e.g. feedback linearization) attempt to strongly change the dynamics of the system often leading to use a high control authority in order to stabilize a linear desired dynamics. Recently, a modelfree damping control approach has been presented in [18] where the kinetic energy stored into the elastic transmission is dissipated by the motor. The approach in [18] is composed of two phases: in the first one the spring is completely loaded (i.e. the link velocity is null) and the reference position is moved towards the link position to stop the link, in the second phase the link is smoothly moved toward the desired position.

In this paper we present a model-free approach for damping control of VSA that takes advantage of the possibility to change the stiffness. Our approach differs from the one presented in [18] because the proposed one i) is derived from optimality conditions for a one DoF system and ii) it does not require to bring the system in the desired position after having dissipated the energy but it allows to set the reference position in the desired position itself. The two 
control strategies are compared with experimental tests in Section VIII.

The Optimal Control (OC) theory has been chosen as the fundamental tool to solve the abovementioned problem. This choice is motivated by the fact that OC is a key element in understanding planning and control methodologies for soft actuators (see e.g. [5], [4] and [19]). A careful analysis of results, obtained through either analytic or numerical techniques, allows to distillate laws summarising control policies that can be applied to different tasks. In this work we first formalize the problem of minimizing the terminal energy for a one DoF spring-mass model in which the stiffness is assumed to be the control input. Afterwards we show through a complete analysis that, under suitable conditions, the optimal control law for the stiffness is bang-bang like and the switches occur when the product of the link speed and the spring deflection changes sign. The rationale of the law is that the stiffness transmission should be maximum when the link is slowing down and getting away from the desired position and minimum when the link is speeding up and going towards the desired position. Therefore, the first result is that the optimal control policy for the stiffness can be summarized in the rule: stiff slow-down and soft speed-up. Notice that the stiffness control law is opposite with respect to the one found in previous works [4] and [20] where the objective was to maximize the link terminal speed. Furthermore we show, through Lyapunov stability theorems, that the same policy can be profitably applied to a multi-DoF system to substantially increase the rate of convergence toward a desired configuration. Finally we validate theoretical results with simulations and experimental tests performed on the qbmoves, a VSA derived by the VSA Cube [10].

A further outcome of this work derives by the comparison of the proposed control policy with human data in an analogous task. For example, in the work [21] it is studied the task of catching a ball with a Kuka LIghtWeight robot where the stiffness is tele-operated by a human. A preliminary analysis of the recorded data, reported in section VI-C, seems to suggest the hypothesis that, at least for the first part of the task, the endpoint stiffness is regulated according to the law found in this study.

\section{Problem Statement}

The dynamic equation of a manipulator with elastic joints is:

$$
\begin{aligned}
M(q) \ddot{q}+C(q, \dot{q}) \dot{q}+G(q) & =f(q-\theta, \sigma)-B \dot{q} \\
I \ddot{\theta}+D \dot{\theta} & =-f(q-\theta, \sigma)+u
\end{aligned}
$$

where $q \in \mathbb{R}^{n}$ is the link configuration vector and $\theta \in \mathbb{R}^{m}$ the motor configuration vector. $M(q) \in \mathbb{R}^{n x n}$ is the inertia matrix of the manipulator, $C(q, \dot{q}) \in \mathbb{R}^{n x n}$ is the centrifugal/Coriolis matrix, $G(q) \in \mathbb{R}^{n}$ the gravity effect and $B \in \mathbb{R}^{n x n}$ the matrix of the viscous friction in the joints. $I \in \mathbb{R}^{m x m}$ and $D \in$ $\mathbb{R}^{n x n}$ are respectively the actuation inertia and the damping matrix. Finally, $u$ is the control input. With $f(q-\theta, \sigma)$ we model the action of the elastic elements that realise the coupling between the motors and the links. In this setting $\sigma$ represents an input for stiffness control. For several VSAs it holds:

$$
f(q-\theta, \gamma)=-K(\gamma)(q-\theta),
$$

where $K(\gamma)=\operatorname{diag}(k(\gamma))$ is the stiffness matrix of the elastic transmissions. For example, in an antagonistic VSA, the model (2) is valid around an equilibrium position, with $\gamma=\frac{\theta_{m, 1}-\theta_{m, 2}}{2}$ and $\theta=\frac{\theta_{m, 1}+\theta_{m, 2}}{2}$, where $\theta_{m, 1}$ and $\theta_{m, 2}$ are the position vectors of the two prime movers of each VSA of the robot. In the following we assume that $f(q-\theta, \gamma)$ has the form given in (2) and that $k(\gamma)$ is the vector control input.

The problem we address in this paper is to find a model-free policy for adjusting the stiffness $k(\gamma)$ for damping control of a variable stiffness robot, the model thereof can be described by the (1).

\section{One DoF VSA: Optimal Control}

From (1), the typical dynamic equation for a single DoF VSA actuator is:

$$
\ddot{q}+w^{2}(q-\theta)=0,
$$

where $q$ is the link position, $\theta$ is the motor position, $w=$ $\sqrt{k / m}$ is the natural frequency of the system, $m$ is the link inertia, and $k$ is the stiffness. It is worth noting that a damping parameter is not considered in (3). On the left of Fig. 1 a representation of the model in exam is reported.

Given a final time $T$, in the following we consider $\theta=$ $\hat{q}, \forall t \in[0, T]$, where $\hat{q}$ represents the desired link terminal position.

Given the state vector $x(t)=\left[x_{1}, x_{2}\right]^{T}=[q-\theta, \dot{q}]^{T} \in \mathbb{R}^{2}$, the control input $u(t)=k \in U=\left[k_{\text {min }}, k_{\text {max }}\right]$ with $k_{\text {min }}$ and $k_{\text {max }}$ minimum and maximum stiffness achievable by the VSA respectively. The system dynamics in state form is:

$$
\dot{x}=f(x, u)=\left[\begin{array}{c}
x_{2}(t) \\
-w^{2} x_{1}(t)
\end{array}\right] .
$$

Having damping effect means to decrease the mechanical energy of the system. This can be efficently obtained by minimizing the following quadratic cost function:

$$
\phi(x(T))=\frac{x_{1}^{2}(T)}{2}+\frac{x_{2}^{2}(T)}{2},
$$

for a fixed terminal time $T$.

Finally, the optimal control problem is:

$$
\begin{aligned}
& \min _{u(t)} \phi(x(T)) \\
& \dot{x}(t)=f(x, u) \\
& x(0)=x_{0} \\
& 0 \leq k_{\text {min }} \leq u(t) \leq k_{\text {max }}
\end{aligned}
$$

where $x_{0}=\left[x_{0,1} x_{0,2}\right]^{T}$ is the vector of the initial conditions. 
The associated Hamiltonian function [22] is then:

$$
H=\lambda^{T} f(x, u)=\lambda_{1}(t) x_{2}(t)-u(t) / m \lambda_{2}(t) x_{1}(t),
$$

where $\lambda=\left[\lambda_{1}, \lambda_{2}\right]^{T} \in \mathbb{R}^{2}$ is the costate vector.

According to the Pontryagin Maximum Principle (PMP) in [22], necessary conditions on the optimal control $u(t)$ can be derived by minimising (5). Since the Hamiltonian is linear on $u(t)$ we can conclude that the optimal control is bang-bang like according to the following law:

$$
u(t)=\left\{\begin{array}{lll}
k_{\max } & \text { when } & \lambda_{2}(t) x_{1}(t)>0, \\
k_{\min } & \text { when } & \lambda_{2}(t) x_{1}(t)<0 .
\end{array}\right.
$$

Hence, the switching function of the Hamiltonian is $\sigma(t)=$ $x_{1}(t) \lambda_{2}(t)$ and a switching instant $t_{s} \in[0, T]$ is such that $\sigma\left(t_{s}\right)=0$.

From the PMP optimality conditions the costate dynamics and terminal conditions are:

$$
\begin{aligned}
\dot{\lambda}(t)^{T} & =-\frac{\partial H(x(t), u(t))}{\partial x(t)}=\left[\begin{array}{c}
w^{2} \lambda_{2}(t) \\
-\lambda_{1}(t)
\end{array}\right] \\
\lambda(T) & =\frac{\partial \phi(x(T))}{\partial x(T)}=\left[\begin{array}{l}
x_{1}(T) \\
x_{2}(T)
\end{array}\right] .
\end{aligned}
$$

Since the stiffness (hence $w$ ) is piecewise constant, see (6), the states and costates dynamics can be integrated in any time interval of amplitude $\Delta t$ between two stiffness consecutive switches, i.e. switching interval. Hence, from (4) and (7a) for $t \in(0, \Delta t)$ we obtain:

$$
\begin{aligned}
& x_{1}(t)=\bar{x}_{1} \cos [w(t-\Delta t)]+\frac{\bar{x}_{2}}{w} \sin [w(t-\Delta t)], \\
& x_{2}(t)=\bar{x}_{2} \cos [w(t-\Delta t)]-\bar{x}_{1} w \sin [w(t-\Delta t)], \\
& \lambda_{1}(t)=\bar{\lambda}_{1} \cos [w(t-\Delta t)]+\bar{\lambda}_{2} w \sin [w(t-\Delta t)], \\
& \lambda_{2}(t)=\bar{\lambda}_{2} \cos [w(t-\Delta t)]-\frac{\bar{\lambda}_{1}}{w} \sin [w(t-\Delta t)],
\end{aligned}
$$

where $\lambda(\Delta t)=\left[\bar{\lambda}_{1}, \bar{\lambda}_{2}\right]^{T}$ and $x(\Delta t)=\left[\bar{x}_{1}, \bar{x}_{2}\right]^{T}$.

The optimal control problem can hence be solved computing the optimal switching laws, i.e. when and under which conditions the control switches from the maximum to the minimum value and vice-versa.

The amplitude $\Delta t$ of a switching interval can be computed considering a generic final state $\left(\bar{x}_{1}, \bar{x}_{2}\right)$, a generic final costate $\left(\bar{\lambda}_{1}, \bar{\lambda}_{2}\right)$, the last switching instant $t_{s}$ (before $\Delta t$ ) and one of the two switching conditions $x_{1}\left(t_{s}\right)=0$ or $\lambda_{2}\left(t_{s}\right)=0$. Without loss of generality we consider the origin $t_{s}$ of $\Delta_{t}$ as $t_{s}=0$. Indeed, from (8), the amplitude of the switching interval:

$$
\begin{aligned}
& \Delta_{t x}=\frac{2}{w_{x}} \arctan \left(-\frac{\bar{x}_{2}}{\bar{x}_{1} w_{x}}+\sqrt{1+\frac{\bar{x}_{2}^{2}}{\bar{x}_{1}^{2} w_{x}^{2}}}\right) \text { for } x_{1}\left(t_{s}\right)=0 \\
& \Delta_{t \lambda}=\frac{2}{w_{\lambda}} \arctan \left(\frac{\bar{\lambda}_{1}}{\bar{\lambda}_{2} w_{\lambda}}+\sqrt{1+\frac{\bar{\lambda}_{1}^{2}}{\bar{\lambda}_{2}^{2} w_{\lambda}^{2}}}\right) \text { for } \lambda_{2}\left(t_{s}\right)=0 .
\end{aligned}
$$

An equivalent but less informative form is:

$$
\begin{aligned}
\Delta_{t x} & =\frac{1}{w_{x}} \arctan \left(\frac{\bar{x}_{1} w_{x}}{\bar{x}_{2}}\right), \\
\Delta_{t \lambda} & =\frac{1}{w_{\lambda}} \arctan \left(-\frac{\bar{\lambda}_{2} w_{\lambda}}{\bar{\lambda}_{1}}\right),
\end{aligned}
$$

where $w_{x}$ is the pulsation of the system in a $\Delta_{t x}$, and $w_{\lambda}$ in a $\Delta_{t \lambda}$ respectively.

By applying condition (7b) in equations (11) and (12), one of the two amplitude is negative based on the sign of the switching function in $T$. Thus, the sign of the final state $x(T)$ influences the condition of the last switch of the sequence before $T$ :

$$
\begin{aligned}
& \text { if } x_{1}(T) x_{2}(T)>0, \text { it holds } x_{1}\left(t_{s}\right)=0, \\
& \text { if } x_{1}(T) x_{2}(T)<0, \text { it holds } \lambda_{2}\left(t_{s}\right)=0 .
\end{aligned}
$$

The influence of final state on the last switching condition will be applied for any switching interval $\Delta t$ to reconstruct all the switches occurrences.

1) Case $\mathbf{x}_{1}(\mathbf{T}) \mathbf{x}_{2}(\mathbf{T})>\mathbf{0}$ : Suppose that after $n$ switches the terminal state verifies $x_{1}(T) x_{2}(T)>0$. From (8) and (13) the state and costate values at the last switching instant, denoted by $t_{s_{n}}$, are:

$$
\begin{array}{ll}
x_{1_{n}}=0 & x_{2_{n}}=\sqrt{x_{2 T}^{2}+x_{1 T}^{2} w_{x}^{2}} \\
\lambda_{1_{n}}=\frac{x_{2 T} x_{1 T}\left(1-w_{x}^{2}\right)}{\sqrt{x_{2 T}^{2}+x_{1 T}^{2} w_{x}^{2}}} & \lambda_{2_{n}}=\frac{x_{2 T}^{2}+x_{1 T}^{2}}{\sqrt{x_{2 T}^{2}+x_{1 T}^{2} w_{x}^{2}}},
\end{array}
$$

where $x\left(t_{s_{n}}\right)=\left[x_{1_{n}}, x_{2_{n}}\right]^{T}, \lambda\left(t_{s_{n}}\right)=\left[\lambda_{1_{n}}, \lambda_{2_{n}}\right]^{T}$ and $w_{x}$ is the pulsation in the switching interval $\left[t_{s_{n}}, T\right]$, i.e., there are no other switches between $t_{s}$ and $T$. It follows that a generic instant of time $t_{i}, t_{s}<t_{i}<T$, in which $x_{1}\left(t_{i}\right)=0$ or $\lambda_{2}\left(t_{i}\right)=0$ cannot exist. Moreover, from (13) and (15), $x_{1}\left(t_{i}\right)$ and $\lambda_{2}\left(t_{i}\right)$ can only be positive and hence, from (6), $w_{x}=\sqrt{\frac{k_{\max }}{m}}$ that corresponds to the maximum control input. Thus, the $n$-th switching variables in (15) are hence univocally determined. It is worth noting that the obtained variables are the final state and costate values of the $n$-1-th switching intervals.

We analize the switching condition at the beginning of the $n-1-$ th switch interval $\left[t_{s_{n-1}}, t_{s_{n}}\right]$.

Consider first the case in which at time $t_{s_{n-1}}$ the switch is caused by $\lambda_{2}\left(t_{s_{n-1}}\right)=0$. From (10) and (15) we have

$$
\Delta_{t \lambda}=\frac{2}{w_{\lambda}} \arctan \left(\alpha+\sqrt{1+\alpha^{2}}\right) .
$$

where $\alpha=\frac{x_{2 T} x_{1 T}\left(1-w_{x}^{2}\right)}{\left(x_{1 T}^{2}+x_{2 T}^{2}\right) w_{\lambda}}$ and $w_{\lambda}=\sqrt{\frac{k_{\min }}{m}}$ is the frequency in the switching interval $\left[t_{s_{n-1}}, t_{s_{n}}\right]$ that corresponds to the minimum control input.

Consider now the case in which at time $t_{s_{n-1}}$ the switch is still caused by $x_{1}\left(t_{s_{n-1}}\right)=0$. From (9) and (15) we have $\Delta_{t x}=\frac{\pi}{w_{x}}$. It can be shown that for any value of $w_{x} \Delta_{t x}>\Delta_{t \lambda}$. Hence, the switch in $t_{s_{n-1}}$ is caused by $\lambda_{2}=0$. 
2) Case $\mathbf{x}_{\mathbf{1}}(\mathbf{T}) \mathbf{x}_{2}(\mathbf{T})<\mathbf{0}$ : The same procedure can be applied for the case $x_{1}(T) x_{2}(T)<0$ where the last switching condition is on $\lambda_{2}$. Considering (8) and (14), for the intervals $\left[t_{s_{n-1}}, t_{s_{n}}\right]$ and $\left[t_{s_{n}}, T\right]$, defined equivalently as the previous case, the state and costate at $t_{s_{n}}$ are:

$$
\begin{array}{ll}
x_{1_{n}}=-\frac{x_{2 T}^{2}+x_{1 T}^{2}}{\sqrt{x_{1 T}^{2}+x_{2 T}^{2} w_{\lambda}^{2}}}, & x_{2_{n}}=\frac{x_{2 T} x_{1 T}\left(w_{\lambda}^{2}-1\right)}{\sqrt{x_{1 T}^{2}+x_{2 T}^{2} w_{\lambda}^{2}}}, \\
\lambda_{1_{n}}=-\sqrt{x_{1 T}^{2}+x_{2 T}^{2} w_{\lambda}^{2}}, & \lambda_{2_{n}}=0 .
\end{array}
$$

Hence, if $x_{1}\left(t_{s_{n-1}}\right)=0$, the switching interval $\left[t_{s_{n-1}}, t_{s_{n}}\right]$ has amplitude:

$$
\Delta_{t x}=\frac{2}{w_{\lambda}} \arctan \left(-\alpha+\sqrt{1+\alpha^{2}}\right),
$$

where $\alpha=\frac{x_{2 T} x_{1 T}\left(1-w_{\lambda}^{2}\right)}{\left(x_{1 T}^{2}+x_{2 T}^{2}\right) w_{x}}$.

On the other hand, in case of $\lambda_{2}\left(t_{s_{n-1}}\right)=0$, the interval for the switching on $\lambda$ is $\Delta_{t \lambda}=\frac{\pi}{w_{\lambda}}$. It can be shown that for any value of $w_{\lambda} \Delta_{t \lambda}>\Delta_{t x}$. Hence, the switch in $t_{s_{n-1}}$ is caused by $x_{1}=0$.

To conclude, the optimal switching sequence is characterized by alternate switching conditions on $\lambda_{2}$ and $x_{1}$. Hence, the initial conditions can be determined as a function of the final states for any number of switches $n$. For space limitations those computations are not herein reported. However, a similar procedure is applied next to a particular case that will be proved to play a crucial role in the definition of a model-free control law.

\section{CASE OF NULl POSITION OR NULl SPEED AT GIVEN FINAL TIME}

We now consider the particular case of minimizing the energy at a given final time $T$ with the additional condition that the desired amount of energy is concentrated in the kinematic or in the elastic potential term, i.e. one of the terminal states value $x(T)$ is zero.

We start considering a generic switching interval $\delta_{1}=$ $\left[0, \Delta t_{1}\right]$ with $x_{1}\left(\Delta t_{1}\right)=\lambda_{1}\left(\Delta t_{1}\right)=0$. Notice that at time $\Delta t_{1}$ the switching condition (13) is verified. For the alternate sequence of switching conditions we have $\lambda_{2}(0)=0$, i.e. the switching condition (14) must be verified at the initial instant of $\delta_{1}$. Hence, from (8d), we obtain $\Delta_{t}=\frac{\pi}{2 w_{\lambda}}$ or $\lambda_{2}\left(\Delta_{t}\right)=0$ where the latter contradicts the alternance of switches.

Moreover, for $\Delta_{t}=\frac{\pi}{2 w_{\lambda}}$, from (8) the initial state and costate values are:

$$
\begin{array}{ll}
x_{1}(0)=-\frac{x_{2}\left(\Delta_{t}\right)}{w_{\lambda}}, & x_{2}(0)=0, \\
\lambda_{1}(0)=-\lambda_{2}\left(\Delta_{t}\right) w_{\lambda}, & \lambda_{2}(0)=0 .
\end{array}
$$

For the second case of null final speed, we consider again a generic switching interval $\delta_{2}=\left[0, \Delta t_{2}\right]$ with $x_{2}\left(\Delta t_{2}\right)=$ $\lambda_{2}(t)=0$. At time $\Delta t_{1}$ the switching condition (14) is verified and hence the switching condition (14) must be verified at the initial instant of $\delta_{2}$, i.e. $x_{1}(0)=0$. From (8a), we obtain
$\Delta t=\frac{\pi}{2 w_{x}}$ or $x_{1}(\Delta t)=0$ where the last condition contradicts the alternance of switches. Hence, from (8) the initial state and costate values are:

$$
\begin{array}{ll}
x_{1}(0)=0, & x_{2}(0)=x_{1}\left(\Delta t_{2}\right) w_{x}, \\
\lambda_{1}(0)=0, & \lambda_{2}(0)=\frac{\lambda_{1}\left(\Delta t_{2}\right)}{w_{x}} .
\end{array}
$$

It is worth noting that the obtained initial conditions for $\delta_{1}$ $\left(\delta_{2}\right)$ coincide with the assumption on the final condition of $\delta_{2}\left(\delta_{1}\right)$. We can then consider an arbitrary number $n$ of sequences of alternate switching intervals $\delta_{1}$ and $\delta_{2}$ depending on the state values at time $T$. Indeed, in case of null final speed or position, the terminal time $T$ coincides with the last switching time $t_{s_{n}}$. Moreover, in case of $x_{1}(T)=0$ we have $\left[t_{s_{n-1}}, T\right]=\delta_{1}$ while if $x_{2}(T)=0$ we have $\left[t_{s_{n-1}}, T\right]=$ $\delta_{2}$.

The state and costate values at a generic switching time $t_{s_{k}}$ can thus be obtained. For the case of null final position and for odd $k$ we have:

$$
\begin{aligned}
& \left(x_{1}\left(t_{s_{k}}\right), x_{2}\left(t_{s_{k}}\right)\right)=\left(-x_{2 T} \frac{w_{x}^{(k-1) / 2}}{w_{\lambda}^{(k+1) / 2}} \sin \left(\frac{k \pi}{2}\right), 0\right), \\
& \left(\lambda_{1}\left(t_{s_{k}}\right), \lambda_{2}\left(t_{s_{k}}\right)\right)=\left(-x_{2 T} \frac{w_{\lambda}^{(k+1) / 2}}{w_{x}^{(k-1) / 2}} \sin \left(\frac{k \pi}{2}\right), 0\right),
\end{aligned}
$$

while for even $k$ :

$$
\begin{aligned}
& \left(x_{1}\left(t_{s_{k}}\right), x_{2}\left(t_{s_{k}}\right)\right)=\left(0, x_{2 T} \frac{w_{x}^{k / 2}}{w_{\lambda}^{k / 2}} \cos \left(\frac{k \pi}{2}\right)\right), \\
& \left(\lambda_{1}\left(t_{s_{k}}\right), \lambda_{2}\left(t_{s_{k}}\right)\right)=\left(0, x_{2 T} \frac{w_{\lambda}^{k / 2}}{w_{x}^{k / 2}} \cos \left(\frac{k \pi}{2}\right)\right) .
\end{aligned}
$$

Given the sequence of $n$ switching intervals, in the $n-k$-th interval the switching function is $\sigma_{k}=x_{1}(t) x_{2}(t) p(k)$ where $p(k)=\left(w_{\lambda} / w_{x}\right)^{k}$ for even $k$ and $p(k)=\left(w_{\lambda} / w_{x}\right)^{k+1}$ for odd $k$.

For the complementary case of null final speed, for odd $k$ we have:

$$
\begin{aligned}
& \left(x_{1}\left(t_{s_{k}}\right), x_{2}\left(t_{s_{k}}\right)\right)=\left(0, x_{1 T} \frac{w_{x}^{(k+1) / 2}}{w_{\lambda}^{(k-1) / 2}} \sin \left(\frac{k \pi}{2}\right)\right), \\
& \left(\lambda_{1}\left(t_{s_{k}}\right), \lambda_{2}\left(t_{s_{k}}\right)\right)=\left(0, x_{1 T} \frac{w_{\lambda}^{(k-1) / 2}}{w_{x}^{(k+1) / 2}} \sin \left(\frac{k \pi}{2}\right)\right),
\end{aligned}
$$

while for even $k$ :

$$
\begin{aligned}
& \left(x_{1}\left(t_{s_{k}}\right), x_{2}\left(t_{s_{k}}\right)\right)=\left(x_{1 T} \frac{w_{x}^{k / 2}}{w_{\lambda}^{k / 2}} \cos \left(\frac{k \pi}{2}\right), 0\right), \\
& \left(\lambda_{1}\left(t_{s_{k}}\right), \lambda_{2}\left(t_{s_{k}}\right)\right)=\left(x_{1 T} \frac{w_{\lambda}^{k / 2}}{w_{x}^{k / 2}} \cos \left(\frac{k \pi}{2}\right), 0\right) .
\end{aligned}
$$

Given the sequence of $n$ switching intervals, in the $n-k$-th interval the switching function is $\sigma_{k}=x_{1}(t) x_{2}(t) r(k)$ where 
$r(0)=p(0)$ and $r(k)=p(k) / w_{x}^{2}$ otherwise. Since $r(k)>0$ the optimal stiffness control policy can be described by

$$
u=\left\{\begin{array}{l}
k_{\max } \text { if }(q-\theta) \dot{q}>0, \\
k_{\min } \text { if }(q-\theta) \dot{q}<0 .
\end{array}\right.
$$

Remark 1: In case of final null position or velocity, the switching function $\sigma$, whose sign determines the optimal switching law, does not depend on the model parameters. Hence, the obtained control law is model-free. On the right of Fig. 1 a scheme of the control law is showed. Moreover, for the considered model the control law in (23) can be written as $u=k_{\max }$ if $\ddot{q} \dot{q}<0$ and $u=k_{\min }$ if $\ddot{q} \dot{q}>0$ that is the opposite of the control law found [4] and [20] where the objective was to maximize the link terminal speed.

\section{Control of A multi DoF VSA Robot}

In this section we apply the model-free stiffness control policy derived in the previous section to the VSA multi DoF robot model described by the equation (1). It is worth to note that in (1) the damping effect is considered with the contribution $-B \dot{q}$. We assume that the motor provides a torque input $u$ such that the motor position $\theta$ is set to bring the robot at the desired equilibrium position $\hat{q}$. In absence of gravity it is $\theta=\hat{q}$. In [18] it is shown that also in presence of gravity a $\theta \neq \hat{q}$ can be found so that $q$ converges to $\hat{q}$. Hence, gravity does not affect the applicability of the proposed controller since it acts only on the stiffness input $k(\sigma)$, and hence from now on in the analysis we consider $\theta=\hat{q}=0$.

In the following we motivate the application of the proposed algorithm through Lyapunov like arguments.

Consider now the state vector $x=[q, \dot{q}]^{T}$, the kinetic energy $T$, the gravitational energy $U_{g}$, and the minimum elastic energy $U_{k_{\min }}=1 / 2(\theta-q)^{T} K_{\min }(\theta-q)$ of the link dynamics described by the first equation of (1) where $K_{\min }=\operatorname{diag}\left(k_{\min }\right)$. The following Lyapunov candidate can be derived:

$V(x)=T+U_{g}+U_{k_{\min }}=\frac{1}{2} \dot{q}^{T} M(q) \dot{q}+U_{g}(q)+U_{k_{\min }}(\theta, q)$.

The time variation of the previous equation is:

$$
\begin{aligned}
\dot{V}(x)=\frac{\partial V}{\partial x} \dot{x}= & \dot{q}^{T} M(q) \ddot{q}+\frac{1}{2} \dot{q}^{T} \dot{M}(q) \dot{q}+G(q)^{T} \dot{q}+ \\
& (q-\theta)^{T} K_{\min }(\dot{q}-\dot{\theta}) .
\end{aligned}
$$

By substituting (1) in (24) and considering that $\dot{M}(q)=$ $2 C(q, \dot{q}) \dot{q}$ it follows:

$$
\dot{V}(x)=-\dot{q}^{T} B \dot{q}-\dot{q}^{T}\left(K(\gamma)-K_{\min }\right)(q-\theta) .
$$

By applying the optimal control policy given in (23) for each component of the control vector $K(\gamma)$, the (25) is negative semi-definite. Nevertheless, since the only trajectory that lays in $R=\{x \mid \dot{V}(x)=0\}$ is zero is the equilibrium, by applying the Krasovskii-Lasalle theorem it is possible to conclude for the asymptotic stability of the origin.

The effect of the control law (23) in the multiple DoF system is to increase the rate of convergence to the equilibrium point since it minimises the (25), i.e. it dissipates as much energy as possible by adjusting the stiffness. It is worth noting that, even though the control law in (23) has been obtained based on the damping-free system (3), in this section it has been shown that it is stabilizing also for systems with viscosities.

\section{Simulations AND EXPERIMENTS}

In this section the simulation for a single DoF robot and the experiments on single and multi DoF VSA are proposed. In Fig. 2 the schemes of the considered system are reported.

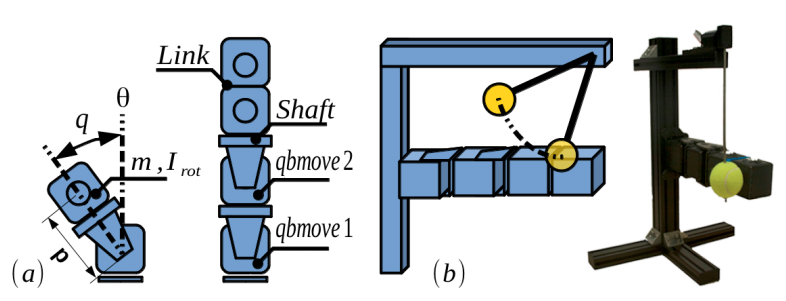

Fig. 2: (a) Scheme of the 1 DoF and 2 DoF planar arms used for the simulations and experiments. The revolute joints are indicated as qbmove 1 and qbmove 2. (b) Experimental setup for the 2 DoF case.

\section{A. Simulation results}

A link of mass $m$ with the center of mass posed at distance $d$ is connected to a VSA which can instantaneously change the stiffness between the motor and the link in the interval $\left[k_{\min }, k_{\max }\right]$. A viscous friction $b$ acts on the joint. Let $\theta$ be the position of the motor, $\hat{q}$ the equilibrium position of the link and $q$ the link position. The actuator dynamic is neglected and gravity doesn't affect the system.

In the simulation the motor position $\theta$ is set equal to the desired link equilibrium position $\hat{q}$. A representation of the model can be observed in the 1 DoF case of in Fig. 2 (a) which is an equivalent implementation of the system presented in Fig. 1. The dynamic parameters of the system are: the mass $m=0.226\left[K_{g}\right]$, the distance of the mass from the center of rotation $d=0.1[\mathrm{~m}]$, the rotational inertia of the link $I_{r o t}=0.001\left[K_{g} m^{2}\right]$, the maximal and minimal stiffness $\left[K_{\max }, K_{\min }\right]=[1,0.3][\mathrm{Nm} / \mathrm{rad}]$ respectively and the module of the viscous friction $b=0.01\left[\mathrm{Nms}^{2} / \mathrm{rad}^{2}\right]$. The link starts from the equilibrium configuration and receives an impulsive external torque of $1 \mathrm{Nm}$ after $0.2 \mathrm{~s}$. In the first and second simulations the actuator is controlled to generate a constant stiffness, at minimum and maximum values respectively. Finally, in the third simulation the stiffness is regulated according to the control law (23). In Fig. 3 the results of the simulations are shown for the three different inputs. The time, from the impact instant, to reach an error position that remains in a neighbourhood of the origin of radius $0.05 \mathrm{rad}$, has been evaluated. Such settling times are indicated in figure with vertical dot lines and their values are: $2.06 \mathrm{~s}$ for the 

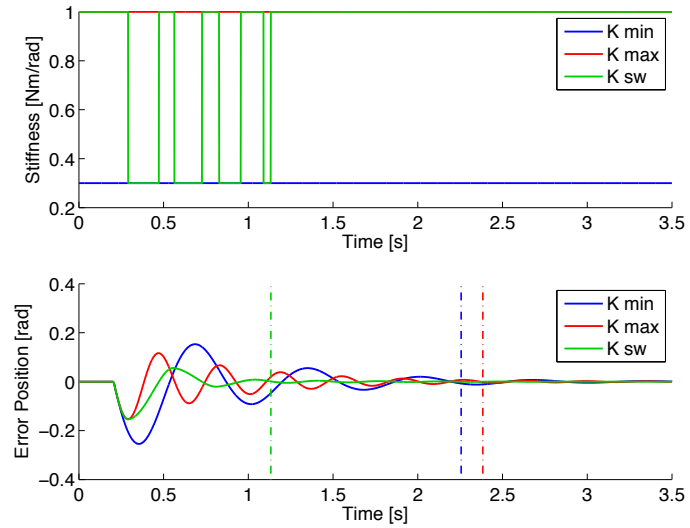

Fig. 3: Top: stiffness evolution under constant minimum and maximum inputs and under the proposed control law. Bottom: evolutions of the link error positions.

minimum stiffness case, $2.18 s$ for the maximum stiffness case, and $0.93 s$ for the optimal control case (where the impact was at $t=0.2 s$ ). In this case, the stiffness switching control produces an improvement of $100 \%$ of the settling time with respect to the constant stiffness cases.

\section{B. Experimental Setup and Implementation}

Two planar robotics arms, 1 DoF and 2 DoF respectively, have been realised with qbmove actuators based on the VSA Cube [10] (see [23] for the datasheet with the main actuators characteristics). The first arm uses another qbmove actuator connected to the first by a shaft as inertia. The second arm uses two qbmove actuators connected to the last active joint by a shaft as inertia. The systems are mounted on a structure to be not influenced by the gravity as shown in Fig. 2. On the top of the structure a pendulum is used to simulate the disturbance, i.e. an impact at the end of the arm.

For the one degree of freedom experiment we compared four different cases: uncontrolled oscillation of the system, PID control based on error position of the link for a constant stiffness reference (half of the range of the qbmove), the bangbang position control presented in [18] with the bring back motion generated by a PID, and the control law obtained with the proposed approach. This has been repeated for two different inertia values. The stiffness control is realised by adjusting the stiffness preset $\gamma=\left(\theta_{m, 1}-\theta_{m, 2}\right) / 2$. This is possible since the control is bang-bang like and the stiffness of the qbmove is a monotonic function of the preset. In Fig. 4 we present a comparison of the link position evolution of the 1 DoF system (with 1-actuator-link) in case of an impact after 1 second under different controls: system at maximum stiffness with null control, PID control, the bangbang position control presented in [18] and the proposed stiffness switching control law. The PID control and the one used for the bring back phase in the bang-bang position control, in [18], have been experimentally tuned. The tuned PID shows the best result in term of settling time which is comparable to the stiffness switching control. In the bottom
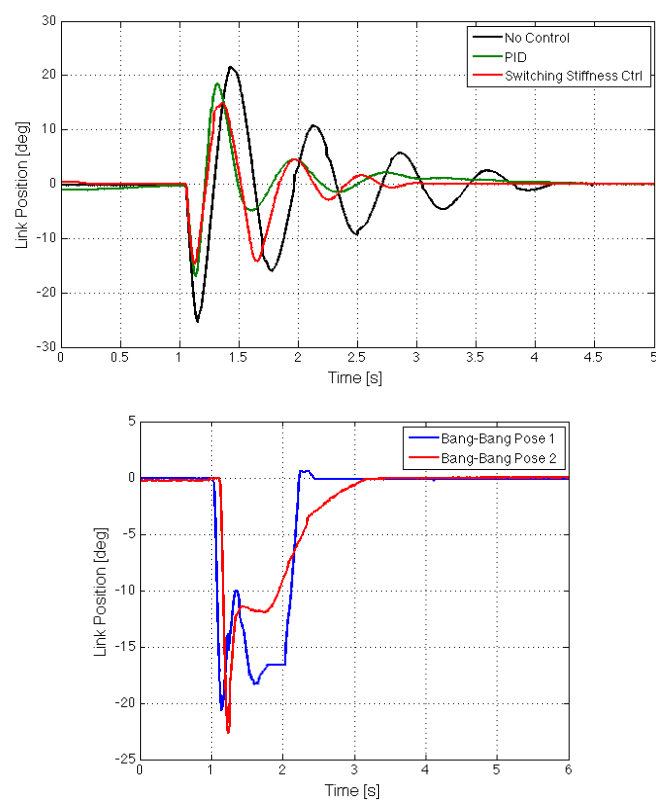

Fig. 4: Top: Link position evolution for different control strategies applied to the $1 \mathrm{DoF}$ system. In red, the evolution in case of the control law proposed in this paper. Bottom: Link position evolution for two different implementation of Bang-Bang position control.

of Fig. 4 two implementation of the Bang-Bang position control are shown. Those controls differ in the timing of the bring back phase and PID. The performance of the control is influenced by the dynamic of the actuator and the control used in the bring back motion.

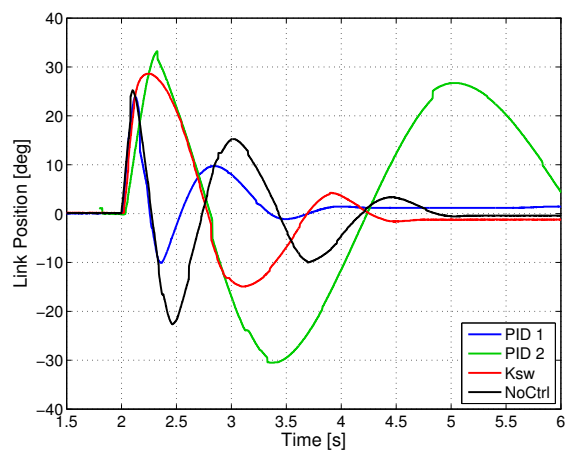

Fig. 5: Link position evolution for different control strategies applied to the 1 DoF system in case of a (almost) double link inertia w.r.t. the case presented in Fig. 4. The PID control presents the best performance but it is tuned on the particular system. The stiffness switching control $K_{S w}$ presents a performance comparable to the performance of PID 1 despite the increased inertia.

In Fig. 5 a further comparison is implemented where the inertia of the link is doubled. The proposed control law is now compared with two other PID controls: the PID1 tuned on the new system characteristics and PID2 is the same 
PID control law used in the previous set of experiments with lower inertia. The stiffness switching control $K_{s w}$ shows robustness to the inertia variation and a performance comparable with the PID1 opportunely tuned. On the other hande, the PID2 shows instability.

Since the qbmove has a stiffness variation time of 0.1 seconds from the minimum to the maximum stiffness values, when the link oscillations happen at a too high frequency the stiffness cannot change instantaneously. For this reason it has been implemented a stiffness control law based on thresholds (experimentally evaluated) on the position and the velocity of the link in order to anticipate the stiffness switchings. A comparison between the theoretical and the threshold-based approach for the one degree of freedom case is reported in Fig. 6. In Fig. 7 and 8 we present the experimental results for the two degrees of freedom case and the comparison between the controlled system (with the law (23)) and the uncontrolled system with low and high stiffness configurations. The comparison shows that the switching control law guarantees an improvement of $25 \%$ of the settling time w.r.t the stiff case.

It is worth noting that in Fig. 6 and 7 the stiffness is measured in $[d e g]$ in the range $[0,35]$ deg that is equivalent to a stiffness values of $[0.3,13] \mathrm{Nm}$ on the output shaft.

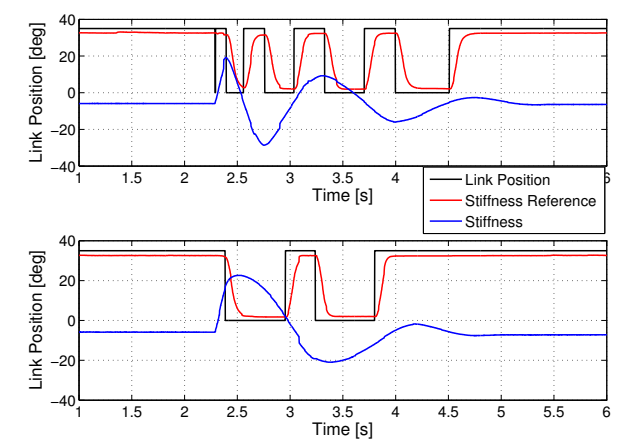

Fig. 6: Link position and preset evolution for the one DoF case with the theoretical control (top) and thresholds-based control (bottom) to anticipate the switching instants

\section{Discussion}

In the following we discuss advantages and drawbacks of the proposed control approach w.r.t. existing controllers.

An advantage is the fact that the stiffness control law does not depend on the model of the system, furthermore it relies on the detection of state events (i.e. zero crossing of deflection and velocity). Furthermore, even though the dynamical system differs from the nominal one the obtained control law can be still applied. While, in this case, optimality cannot be guaranteed anymore, the Lyapunov based analysis proves that using the control law in case of damping at the link side the settling time of the system is improved. Moreover the proposed approach can be profitably used to stabilise a multidof VSA robot. These characteristics, belonging also
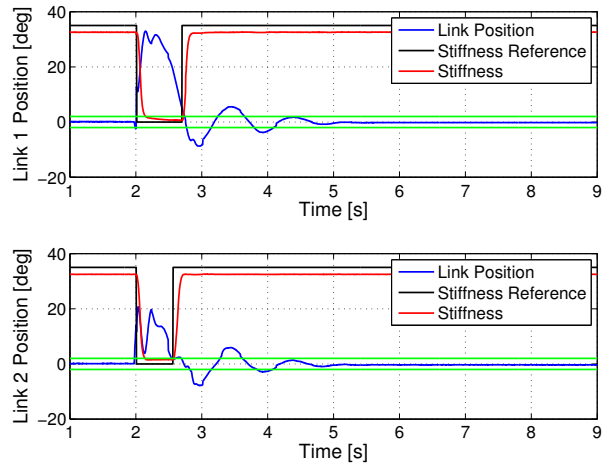

Fig. 7: Links position evolution for the stiffness switching control applied to the two DoF case. The reference switchings of the preset are reported in black while the measured preset is in red. The settling time from the impact to reach the desired position with an error of $\pm 2 \mathrm{deg}$ (in green) is $T_{s w 1}=2.098 s$ and $T_{s w 2}=2.078 s$ for the first and second link respectively.
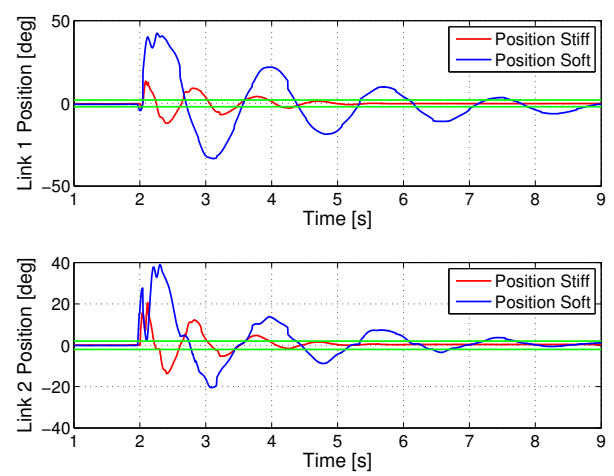

Fig. 8: Links position evolution for the uncontrolled 2 DoF system with constant preset. The evolution of the links after the impact in case of maximum preset are indicated in red, while in case with minimum preset are indicated in blue. The settling times in the stiff case are $T_{\text {stiff }}=2.438 \mathrm{~s}, T_{\text {stiff }}=$ $2.032 s$ for first and second link respectively, while for the soft case $T_{\text {soft }}>8 s, T_{\text {soft }}=5.738 \mathrm{~s}$.

to the controller presented in [18], make it relatively easily applicable to a variety of systems since it does not requires a carefully identified model. A difference w.r.t. the law found in [18] is that the stiffness switching control law does not require a further control to bring the system to the desired position.

An important limitation is that if the stiffness regulation time is limited, the controller performance decays and the stiffness switchings must be anticipated to achieve better damping performances (see Fig. 6). Since the controller does not take into account the inertia coupling a model-based controller (provided with an accurate model) can have higher performance. 
A further approach to control VSA robots that has been proposed in recent years is the tele-impedance. In this approach the stiffness regulation of the robot is tele-operated by a human being. In [21] it has been studied the task of catching a ball with a Kuka LIghtWeight robot where the stiffness is regulated with the tele-impedance approach. The reported data (Fig. 6 Tele-Impedance and Fig.7 End Point Stiffness in section IV Results in [21] and in video https:// www youtube. $\mathrm{com} /$ watch? $\mathrm{v}=-\mathrm{Fn} 2 \mathrm{dObnFpw}$ ) present a peak of End Point stiffness at the beginning of the task. The temporal evolution of the stiffness and error position of the end effector seems to suggest that the stiffness is increased when the end effector of the robot is getting away from the desired position and it is decreased when the end effector is coming back toward the desired position. This is similar to the behaviour of the system under the control policy presented in this paper. However, further studies are required to drawn proper conclusions.

\section{CONCLUSIONS}

In this paper a model-free approach for damping control of VSA robots through stiffness variation has been proposed. The stiffness control law has been derived by analytically solving the optimal control problem of minimizing the terminal energy for a one DoF spring-mass model with controlled stiffness. The stiffness switching control law uses a maximum stiffness when the link gets away from the desired position and a minimum one when the link is going towards it. A preliminary analysis of data presented in the literature seems to suggest that the proposed law could explain how humans change their stiffness for oscillation damping. Based on Lyapunov stability theorems the obtained law has been proved to be stable for a multi-DoF system. Simulations and experimental tests that validate the theoretical results have been reported.

\section{ACKNOWLEDGMENTS}

The authors would like to thank Kamilo Melo and Hamal Marino for the support and the discussions in the preliminary phase of this work.

\section{REFERENCES}

[1] A. Albu-Schaffer, O. Eiberger, M. Grebenstein, S. Haddadin, C. Ott, T. Wimbock, S. Wolf, and G. Hirzinger, "Soft robotics," IEEE Robotics \& Automation Magazine, vol. 15, pp. 20-30, 2008.

[2] A. Velasco, G. M. Gasparri, M. Garabini, L. Malagia, P. Salaris, and A. Bicchi, "Soft-actuators in cyclic motion: Analytical optimization of stiffness and pre-load," in IEEE-RAS International Conference on Humanoid Robots, 2013.

[3] M. Uemura and S. Kawamura, "Resonance-based motion control method for multi-joint robot through combining stiffness adaptation and iterative learning control," in Proceedings of the IEEE international conference on Robotics and Automation, 2009, pp. 3398-3403.

[4] M. Garabini, A. Passaglia, F. Belo, P. Salaris, and A. Bicchi, "Optimality principles in variable stiffness control: The vsa hammer," in Intelligent Robots and Systems (IROS), 2011 IEEE/RSJ International Conference on, Sept 2011, pp. 3770-3775.
[5] S. Haddadin, M. Weis, S. Wolf, and A. Albu-Schäffer, "Optimal control for maximizing link velocity of robotic variable stiffness joints," in IFAC World Congress, 2011, pp. 6863-6871.

[6] M. H. David J. Braun and S. Vijayakumar, "Exploiting variable stiffness in explosive movement tasks," Robotics: Science and Systems, 2011.

[7] R. Ham, T. Sugar, B. Vanderborght, K. Hollander, and D. Lefeber, "Compliant actuator designs," Robotics Automation Magazine, IEEE, vol. 16, no. 3, pp. 81 -94, september 2009.

[8] B. Vanderborght, R. Van Ham, D. Lefeber, T. G. Sugar, and K. W. Hollander, "Comparison of mechanical design and energy consumption of adaptable, passive-compliant actuators," Int. J. Rob. Res., vol. 28, pp. 90-103, January 2009.

[9] G. A. Pratt and M. Williamson, "Series elastics actuators," in IROS, 1995, pp. 399-406.

[10] M. G. Catalano, G. Grioli, M. Garabini, F. Bonomo, M. Mancini, N. Tsagarakis, and A. Bicchi, "Vsa - cubebot. a modular variable stiffness platform for multi degrees of freedom systems," 2011.

[11] B. Vanderborght, A. Albu-Schaeffer, A. Bicchi, E. Burdet, D. G. Caldwell, R. Carloni, M. G. Catalano, G. Ganesh, M. Garabini, G. Grioli, S. Haddadin, A. Jafari, M. Laffranchi, D. Lefeber, F. Petit, S. Stramigioli, N. G. Tsagarakis, M. V. Damme, R. V. Ham, L. C. Visser, and S. Wolf, "Variable impedance actuators: Moving the robots of tomorrow," International Conference of Intelligent Robots and Systems - IROS - Best Jubilee Video Award, 2012.

[12] M. Grebenstein, A. Albu-Schäffer, T. Bahls, M. Chalon, O. Eiberger, W. Friedl, R. Gruber, S. Haddadin, U. Hagn, R. Haslinger et al., "The dlr hand arm system."

[13] M. Laffranchi, N. G. Tsagarakis, and D. G. Caldwell, "Compacttm arm: a compliant manipulator with intrinsic variable physical damping," Robotics, p. 225, 2013.

[14] G. Palli, C. Melchiorri, and A. De Luca, "On the feedback linearization of robots with variable joint stiffness," in Robotics and Automation, (ICRA). IEEE International Conference on, 2008, pp. 1753-1759.

[15] I. Sardellitti, G. A. Medrano-Cerda, N. Tsagarakis, A. Jafari, and D. G. Caldwell, "Gain scheduling control for a class of variable stiffness actuators based on lever mechanisms," Robotics, IEEE Transactions on, vol. 29, no. 3, pp. 791-798, 2013.

[16] F. Miyazaki, S. Kawamura, M. Matsumori, and S. Arimoto, "Learning control scheme for a class of robot systems with elasticity," in Decision and Control, 25th IEEE Conference on, vol. 25, 1986, pp. 74-79.

[17] F. Petit and A. Albu-Schaffer, "State feedback damping control for a multi dof variable stiffness robot arm," in Robotics and Automation (ICRA), IEEE International Conference on, 2011, pp. 5561-5567.

[18] F. Petit, C. Ott, and A. Albu-Schaffer, "A model free approach to vibration suppression for intrinsically elastic robots," in Robotics and Automation (ICRA), IEEE International Conference on, 2014, pp. 2176-2182.

[19] A. Bicchi and G. Tonietti, "Fast and soft arm tactics: Dealing with the safety-performance trade-off in robot arms design and control," IEEE Robotics and Automation Magazine, vol. 11, no. 2, p. 22-33, 2004.

[20] M. Garabini, A. Passaglia, F. Belo, P. Salaris, and A. Bicchi, "Optimality principles in stiffness control: The vsa kick," in Robotics and Automation (ICRA), IEEE International Conference on, 2012, pp. 3341-3346.

[21] A. Ajoudani, N. G. Tsagarakis, and A. Bicchi, "Tele-impedance: Towards transferring human impedance regulation skills to robots," in International Conference of Robotics and Automation - ICRA, Saint Paul, MN, USA, May 14 - 18 2012, pp. 382 - 388.

[22] L. S. Pontryagin, Mathematical theory of optimal processes. CRC Press, 1987.

[23] qb robotics s.r.l. (2013). [Online]. Available: http://www.qbrobotics. com/QB_Robotics/PreHome.html 